\title{
PROGNOSE DA PRODUÇÃO DE Eucalyptus camaldulensis DEHNH. PELA APLICAÇÃO DA FUNÇÃO DE DISTRIBUIÇÃO $S_{B}$ DE JOHNSON ${ }^{1}$
}

\author{
Versides Sebastião de Moraes e Silva ${ }^{2}$, Thelma Shirlen Soares ${ }^{3}$, Chirle Colpini², Danielle Parra \\ Travagin ${ }^{2}$, Roberto Tuyoshi Hosokawa ${ }^{4}$ José Roberto Soares Scolforo ${ }^{5}$
}

\begin{abstract}
RESUMO - O objetivo deste estudo foi realizar a prognose da produção de um povoamento de Eucalyptus camaldulensis Delnh, localizado em Cuiabá, MT. Na distribuição diamétrica, utilizou-se a Função $S_{\mathrm{B}}$ de Johnson ajustada pelo método dos momentos. O modelo testado para expressar os atributos da floresta foi avaliado por meio de análise de regressão. De maneira geral, com os testes realizados foi possível verificar que o modelo apresentou ajuste satisfatório e sem tendência nos resíduos. A eficiência de prognose foi avaliada pelo teste "t", desvio de prognose e correlação entre o volume prognosticado e o volume observado na idade de prognose. O processo de modelagem utilizado permitiu obter, com detalhes, as análises das tendências do crescimento, a partir das quais se pode concluir que a metodologia adotada permitiu a obtenção de estimativas da produção atual e futura, utilizando-se de um conjunto de modelos biomatemáticos discriminados em cada fase deste estudo.
\end{abstract}

Palavras-chave: Funções de densidade de probabilidade, classes diamétricas, projeção e predição.

\section{Eucalyptus camaldulensis DEHNH. YIELD PROJECTION USING JOHNSON $S_{B}$ DISTRIBUTION}

\begin{abstract}
This study was carried out to accomplish the yield projection of Eucalyptus camaldulensis Delnh. in Cuiabá-MT. For the diameter distribution, the Johnson SB's function was adjusted by the methods of the moments. The model used to express the attributes of the forest was evaluated by the regression analysis. In general, the tests carried out showed that the model presented satisfactory fitting and was unbiased in the graphic analyses of the residues. The efficiency of the prognosis was appraised by the t-test, prognosis deviation and correlation between the predicted and the observed volume at the same age. The modeling process used allowed us to obtain the detailed tendencies of growth. From these tendencies, it can be concluded that the adopted methodology allowed us to obtain the estimates of the current or future production, making use of the group of bio-mathematical models discriminated for each phase of this study.
\end{abstract}

Keywords: Probability density functions, diameter classes, projection and prediction.

\section{INTRODUÇÃO}

A escassez de matéria-prima aliada ao aumento de consumo e, ainda, somado à política de reposição florestal na Amazônia brasileira tem provocado aumento do interesse de algumas empresas em investir na formação de florestas com espécies de rápido crescimento, com a finalidade de atender à sua própria demanda e à do mercado.
A necessidade de maiores conhecimentos sobre o comportamento das espécies de rápido crescimento tem levado esses empresários a recorrer à busca de informações técnicas que satisfaçam as suas necessidades. Para atender a essa demanda de informações técnicas sobre o comportamento de espécies exóticas e nativas em condições de reflorestamento, ela se tornou uma das mais

\footnotetext{
${ }^{1}$ Recebido em 29-11-2007 e aceito para publicação em 23.06.2009.

${ }^{2}$ Universidade Federal de Mato Grosso, Departamento de Engenharia Florestal - Cuiabá, MT. E-mail:<versides@ufmt.br>.

${ }^{3}$ Universidade Federal dos Vales do Jequitinhonha e Mucuri, Departamento de Engenharia Florestal - Diamantina, MG. E-mail:<thelma.soares@ufvjm.edu.br>.

${ }^{4}$ Universidade Federal do Paraná, Departamento de Ciências Florestais - Curitiba, PR - Brasil. E-mail: <hosokawa@ floresta.ufpr.br>.

${ }^{5}$ Universidade Federal de Lavras, Departamento de Ciências Florestais - Lavras, MG - Brasil. E-mail: <scolforo@ufla.br>.
} 
importantes e urgentes questões a serem pesquisadas e que devem ser aplicadas ao desenvolvimento florestal da região.

Em Mato Grosso, os reflorestamentos são na ordem de 140.000 ha (SHIMIZU et al., 2007), sendo os principais reflorestamentos formados com as espécies de Eucalyptus spp. e Tectona grandis L.f., e esta última tem sido mais amplamente plantada em várias regiões, principalmente no Centro, Noroeste e Norte do Estado. Entretanto, ainda são escassos os programas de pesquisa visando estudar e monitorar o crescimento e incremento dessas florestas, bem como desenvolver um sistema de predição de análise da produção presente e futura, com o propósito de definir as melhores condições de plantio e sítio que permitam quantificar o melhor rendimento dessas espécies, com o firme propósito de auxiliar as tomadas de decisão no gerenciamento e manejo dessas áreas florestadas.

O objetivo deste estudo foi prognosticar a produção presente e futura de um povoamento de Eucalyptus camaldulensis Dehnh. pela aplicação da função de distribuição $S_{B}$ de Johnson.

\section{MATERIAL E MÉTODOS}

\subsection{Fonte de dados}

Este estudo foi desenvolvido com dados da primeira rotação de povoamentos de Eucalyptus camaldulensis Denhn., plantados no espaçamento 3,0 m x 2,0 m, localizados em Cuiabá, Estado de Mato Grosso.

Foram realizados inventários em 530 parcelas permanentes, sendo 53 de $250 \mathrm{~m}^{2}, 105$ de $600 \mathrm{~m}^{2}$ e 371 de $700 \mathrm{~m}^{2}$, durante um período de cinco anos consecutivos, considerando-se a representação de cada parcela por hectare. Todas as parcelas utilizadas tiveram pelo menos duas medições, em que foram mensurados os diâmetros a 1,3 m de altura do solo (dap) de todas as árvores e a altura total (Ht). No caso das alturas, foram medidas em linhas alternadas de cada parcela, sendo medidas 20, 50 e 60 árvores/parcelas nas parcelas de $250 \mathrm{~m}^{2}, 600 \mathrm{~m}^{2}$ e $720 \mathrm{~m}^{2}$, respectivamente. As demais alturas foram estimadas por equações de relações hipsométricas.

As parcelas tiveram suas medições iniciadas aos 17 meses de idade e foram remedidas, em alguns casos, até 104 meses em diversas condições de sítio. Foram distribuídos sistematicamente na área na proporção de uma parcela amostral em cada 10 ha. As parcelas amostrais foram estratificadas de acordo com cada projeto e idade, como preconizou Péllico Netto e Brena (1983), e, posteriormente, foram processados para obter os valores médios de cada variável por parcela em cada período de medição.

As estimativas volumétricas de árvores individuais, basearam-se na cubagem rigorosa de 546 árvores, obtidas ao longo de todo o período de observação, as quais cobriram toda amplitude de idade e, também, as diferentes classes de diâmetro.

Foi realizada a classificação da qualidade do local por meio do método da diferença de equações, em que se tornou possível classificar as parcelas amostradas por índice de sítio a partir do conhecimento da altura dominante encontrada por ocasião dos levantamentos. A partir das equações obtidas, foram estabelecidas três classes de produtividade, estabelecendo-se a idade de referência de 84 meses, em função da máxima idade, que foi de 104 meses. As classes de produtividade variam entre o valor da altura dominante na idade de referência em três desvios-padrão, resultando no intervalo com amplitude que variou de um mínimo de $11,66 \mathrm{~m}$ até $20,37 \mathrm{~m}$ de altura.

\subsection{Sistema de prognose}

O sistema de prognose é viabilizado pela utilização de uma função de distribuição e dos os atributos do povoamento. Procurou-se selecionar os atributos mais correlacionados com os parâmetros das funções de distribuição. Fez-se, então, estimativa dos parâmetros da distribuição na idade desejada da prognose.

Os atributos do povoamento foram estudados com a apreciação de modelos matemáticos que melhor descreveram a evolução de cada variável com a idade e o sítio. Para estimar os atributos do povoamento, foram ajustados e selecionados modelos, entre vários existentes na literatura florestal, para: diâmetro mínimo, área basal, altura, diâmetro máximo, variância dos diâmetros, diâmetro médio quadrático, média aritmética dos diâmetros e sobrevivência de árvores (LEITE, 1990; CUNHA NETO et al., 1994; GUIMARÃES, 1994; SCOLFORO e MACHADO, 1996; OLIVEIRA et al., 1998; SILVA, 1999, entre outros).

O critério de validação da equação mais precisa foi o coeficiente de determinação $\left(R^{2}\right)$, erro-padrão residual $\left(\mathrm{S}_{\mathrm{yx}}\right)$ e distribuição gráfica dos resíduos. 
A função de distribuição de probabilidade (fdp) empregada foi a $S_{B}$ de Johnson:

$\mathrm{f}\left(\mathrm{d}_{\mathrm{i}}, \varepsilon, \lambda, \delta, \gamma\right)=\frac{\delta}{\sqrt{2 \pi}} \frac{\lambda}{\left(\mathrm{d}_{\mathrm{i}}-\varepsilon\right)\left(\lambda+\varepsilon-\mathrm{d}_{\mathrm{i}}\right)} \exp \left\{-1 / 2\left[\gamma+\delta \ln \left[\frac{\mathrm{d}_{\mathrm{i}}-\varepsilon}{\lambda+\varepsilon-\mathrm{d}_{\mathrm{i}}}\right]\right]^{2}\right\}$

Para $\varepsilon<\delta_{1}<\varepsilon<\lambda ;-\infty<\varepsilon<+\infty \varepsilon \lambda>0 \varepsilon-\infty<$ $\gamma<+\infty, \varepsilon \delta>0$

em que:

$\delta_{1}=$ diâmetro da iésima árvore da parcela;

$\varepsilon=$ parâmetro de locação;

$\lambda=$ parâmetro de escala; e

$\delta \varepsilon \gamma=$ determinam a forma da distribuição, em que d é o parâmetro curtose, e g representa a assimetria.

A estimativa dos parâmetros dessa função de densidade de probabilidade (fdp) foi feita pelo método dos momentos. O ajuste das equações dos parâmetros foi efetuado por regressão pelo método "stepwise", e os critérios de validação foram os mesmos empregados na função $S_{\text {в }}$ de Johnsosn.

O parâmetro locação $(\varepsilon)$ foi vinculado a um percentual do diâmetro mínimo de $35 \%$, buscando obter a produção futura em volume, número de árvores e da área basal por parcelas na idade futura, comparando com os resultados obtidos na floresta por ocasião do inventário.

\subsection{Teste de eficiência}

Foram efetuadas três modalidades para avaliar o desempenho do sistema de prognose de cada distribuição utilizada pelos diferentes métodos de ajustes. Em cada teste, tomou-se como base os dados da primeira medição, e prognosticou-se o volume para a idade na qual as parcelas foram remedidas nas diversas ocasiões. Comparou-se o volume das parcelas obtidas na ocasião do inventário, que foi tomado como observado, com o volume obtido por meio do sistema de prognose como estimado.

A avaliação dos resultados foi realizada por meio do teste " $t$ " pareado da diferença entre valor estimado pelo sistema na idade de prognose e o valor observado na ocasião das remedições. Os testes foram realizados para a floresta estratificada por classes de idade e produtividade.

\section{RESULTADOS E DISCUSSÕES}

\subsection{Atributos do povoamento}

$\mathrm{Na}$ Tabela 1 são apresentadas as equações selecionadas para o povoamento em estudo. Parte dessas equações permite efetuar a prognose dos atributos do povoamento desde que sejam fornecidas características do povoamento mensuradas nas parcelas do inventário florestal. O outro conjunto de equações permite obter a prognose dos atributos do povoamento.

\subsection{Funções dos Parâmetros de SB, de Johnson}

Na utilização da função de probabilidade de distribuição $S_{B}$ foi necessário ajustar nos modelos matemáticos que permitem expressar a evolução da média transformada $\left(M_{f i}\right)$, do desvio transformado $\left(D_{f i}\right)$, dos parâmetros curtose (d) e assimetria (g) da função.

As equações que obtiveram melhor performance para os parâmetros da função encontram-se relacionadas na Tabela 2.

De acordo com os resultados do ajuste $(\mathrm{R})$ da média transformada $\left(\mathrm{M}_{\mathrm{fi}}\right)$, pode-se observar que o modelo apresentou um ajuste de $\mathrm{R}^{2}=0,967$ e um baixo resíduo absoluto $\mathrm{S}_{\mathrm{yx}}=0,0434$. Entretanto, quando se faz a transformação do erro absoluto para percentual, o modelo demonstra ser instável para os valores que se aproximam de zero. Essa instabilidade causa um erro percentual muito elevado. Esse mesmo efeito foi observado por Scolforo e Thiersch (1998), em estudo em plantações de Eucalyptus camaldulensis Dehnh., no Estado de Minas Gerais.

A título de exemplo, pode-se citar, quando o valor da média transformada na parcela foi de $-0,03$ e o valor estimado de 0,04, houve erro percentual de 239,66\%. Essa situação mostra que, para valores próximos de zero a função que expressa a média transformada é muito sensível e fortemente influenciada pela transformação logarítmica dos dados.

À medida que o modelo se afasta de zero, ele é minimizado, tornando-se mais estável e produzindo distribuição mais regular, o que ocorre a partir dos valores $\pm 0,20$. Já, quando o gráfico dos resíduos é analisado para os erros absolutos, a situação anterior não acontece. Entretanto, é bom ressaltar que esses efeitos não prejudicam a aplicação do modelo, visto que esses erros absolutos são pequenos.

R. Árvore, Viçosa-MG, v.33, n.5, p.853-863, 2009 
As figuras 1A e 1B ilustram melhor o comportamento da função que explica a evolução da média transformada em termos absolutos e em porcentagem. O modelo que estima a evolução dos desvios transformados da função $\mathrm{S}_{\mathrm{B}}$ demonstra ser eficiente com ajuste $\mathrm{R}^{2}=0,98$ e uma precisão $\mathrm{S}_{\mathrm{yx}} \%=1,96$. Os desvios ao longo do eixo foram sempre menores que $10 \%$, o que garante boa estimativa. Observou-se, nesse caso, que a transformação logarítmica aplicada aos dados não provocou o mesmo efeito ocorrido na média transformada. Na mesma Figura $1 \mathrm{C}$, pode-se observar o comportamento gráfico da distribuição dos resíduos em porcentagem dos desvios transformados.

A função que expressa a evolução do parâmetro curtose (d) da função $S_{B}$ apresenta comportamento que pode ser considerado adequado para as condições estatísticas de formatação de modelos. Com um ajuste $\operatorname{de} \mathrm{R}^{2}=0,9786$ e precisão em porcentagem $\left(\mathrm{S}_{\mathrm{yx}} \%=2,0224\right)$ mais os resíduos normalmente distribuídos ao longo do eixo da curtose da função $S_{B}$, pode-se afirmar que o modelo está dentro dos limites estatisticamente aceitáveis para esses critérios de avaliação. Convém ressaltar que, para esse parâmetro, o efeito da logaritmização não provocou mudanças no comportamento da distribuição dos resíduos. A Figura 1D ilustra melhor o comportamento da distribuição dos resíduos em porcentagem do parâmetro curtose.

A equação que expressa a evolução do parâmetro assimetria ( $\mathrm{g}$ ) da função $\mathrm{S}_{\mathrm{B}}$ demonstra que o conjunto de dados apresenta assimetria negativa, pois a sua média é de -0,2087, e, dessa forma, a distribuição do erro em porcentagem não se apresenta de forma satisfatória, provocando inclinação ao longo do eixo de simetria, como pode ser visto na Figura 1E. Entretanto, convém ressaltar que o modelo, quando avaliado pelo ajuste, é considerado satisfatório, pois o resultado

Tabela 1 -Estimativa dos parâmetros, coeficiente de determinação $\left(\mathrm{R}^{2}\right)$, valor F, erro-padrão residual (EPR) e coeficiente de variação (CV\%)

Table 1 - Parameter estimates, determination coefficient $\left(\mathrm{R}^{2}\right)$, F value, residual standard error (EPR) and variation coefficient (CV\%).

\begin{tabular}{|c|c|c|c|c|c|c|}
\hline Atributo & Equação* & $\mathrm{R}^{2}$ & $\mathrm{R}_{\mathrm{aj}}^{2}$ & $F$ & EPR & CV\% \\
\hline $\begin{array}{l}\text { Diâmetro } \\
\text { minimo }\end{array}$ & $\begin{array}{l}\mathrm{D}_{\min }=-0,10322+0,00008 \mathrm{I}^{2}-0,33158 \\
\ln \left(\mathrm{N}_{\mathrm{ha}}\right)+0,73686 \ln \left(\mathrm{D}_{\mathrm{g}}\right)+1,89503 \\
\ln \left(\mathrm{H}_{\mathrm{dom}}\right)-1,15070\left(\frac{\mathrm{H}_{\mathrm{dom}}}{\mathrm{I}}\right)\end{array}$ & 0,94 & 0,94 & 997,3 & 0,31 & 9,18 \\
\hline Área basal & $\begin{array}{l}\mathrm{AB}=-3,20061+1,99991 \ln \left(\mathrm{D}_{\mathrm{m}}\right)+ \\
0,00076\left(\mathrm{~N}_{\mathrm{ha}}-\mathrm{D}_{\max }\right)\end{array}$ & 0,99 & 0,99 & 20782,4 & 0,22 & 3,20 \\
\hline Altura & $\begin{array}{l}\ln \left(\mathrm{H}_{\mathrm{CLD}}\right)=1,25375+0,00891 \mathrm{D}_{\mathrm{i}}+ \\
0,11967 \ln (\mathrm{Dg} \times \mathrm{I})+0,04266 \mathrm{H}_{\mathrm{dom}}- \\
0,36848\left(\frac{\mathrm{D}_{\mathrm{g}}}{\mathrm{D}_{\mathrm{i}}}\right)+0,08165 \ln (\mathrm{AB})\end{array}$ & 0,91 & 0,91 & 6353,4 & 0,08 & 3,61 \\
\hline $\begin{array}{l}\text { Diâmetro } \\
\text { máximo }\end{array}$ & $\begin{array}{l}\mathrm{D}_{\max }=0,68529+1,22179 \mathrm{H}_{\mathrm{dom}}+ \\
0,04019 \mathrm{H}_{\text {dom }}^{2}+0,0036 \mathrm{I}^{2}- \\
7,46115\left(\frac{\mathrm{N}_{\text {ha }}}{\mathrm{I}^{3}}\right)\end{array}$ & 0,91 & 0,91 & 739,3 & 1,41 & 10,39 \\
\hline $\begin{array}{l}\text { Variância } \\
\text { dos diâmetros }\end{array}$ & $\begin{array}{l}\ln \left(\mathrm{s}^{2}\right)=1,44709+0,59798 \ln \left(\mathrm{D}_{\text {max }}\right)- \\
0,17105 \ln \left(\mathrm{N}_{\mathrm{ha}}\right)+0,86492 \ln \left(\mathrm{D}_{\mathrm{g}}-\mathrm{D}_{\mathrm{m}}\right)+ \\
0,33557 \ln \left(\mathrm{H}_{\mathrm{dom}}\right)\end{array}$ & 0,97 & 0,97 & 3092,5 & 0,44 & 8,34 \\
\hline $\begin{array}{l}\text { Diâmetro } \\
\text { médio } \\
\text { quadrático }\end{array}$ & $\begin{array}{l}\mathrm{D}_{\mathrm{g}}=0,036345+0,03160 \mathrm{H}_{\mathrm{dom}}+0,70064 \\
\mathrm{D}_{\mathrm{m}} x\left(1+0,40494 \exp \left(-0,67413 \mathrm{I}^{-0,92371}\right)\right)\end{array}$ & 0,99 & 0,99 & 0,1 & 1,11 & 0,99 \\
\hline $\begin{array}{l}\text { Média } \\
\text { aritmética } \\
\text { dos diâmetros }\end{array}$ & 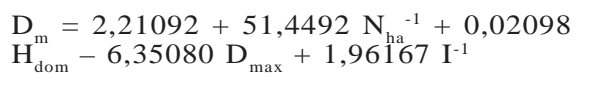 & 0,79 & 0,78 & 484,8 & 0,59 & 7,35 \\
\hline
\end{tabular}

Em que: $\mathrm{D}_{\min }=$ diâmetro mínimo aproveitável $(\mathrm{cm}) ; \mathrm{I}=$ idade (meses); $\mathrm{N}_{\mathrm{ha}}=$ número de árvores por hectare; $\mathrm{H}_{\mathrm{dom}}=$ altura dominante $(\mathrm{m}) ; \mathrm{AB}=$ área basal $\left(\mathrm{m}^{2} / \mathrm{ha}\right) ; \mathrm{D}_{\mathrm{m}}=$ média aritmética do diâmetro $(\mathrm{cm}) ; \mathrm{D}_{\max }=$ diâmetro máximo $(\mathrm{cm}) ; \mathrm{H}_{\mathrm{CLD}}$ $=$ altura total das árvores nas classes de diâmetro $\mathrm{i}(\mathrm{m}) ; \mathrm{D}_{\mathrm{i}}=$ valor do centro da classe de diâmetro $(\mathrm{cm}) ; \mathrm{s}^{2}{ }_{\mathrm{D}}=$ variância dos diâmetros; e $\mathrm{D}_{\mathrm{g}}=$ diâmetro médio quadrático $(\mathrm{cm})$.

R. Árvore, Viçosa-MG, v.33, n.5, p.853-863, 2009 
obtido foi de 0,9883 , enquanto o erro-padrão absoluto de assimetria foi de 0,0365 . Quando é avaliado sob os aspectos de valores absolutos, tanto as estimativas do parâmetro quanto a distribuição dos resíduos apresentam-se, de forma adequada, para incorporar o sistema de prognose. Já a Figura 1F mostra o comportamento gráfico dos resíduos absolutos do modelo que expressa a evolução da assimetria da função $S_{B}$.

\subsection{Prognose}

A prognose, quando aplicada no total da floresta e considerando individualmente cada uma das parcelas, demonstrou ser instável na projeção das variáveis volume, número de árvores e área basal. Os desvios, quando avaliados das menores para as maiores idades, ficaram fora dos limites estatisticamente aceitáveis, tornando-se necessária a estratificação da floresta.

Tabela 2 -Estimativa dos parâmetros, coeficiente de determinação $\left(\mathrm{R}^{2}\right)$, valor F, erro-padrão residual (EPR) e coeficiente de variação (CV\%).

Table 2 - Parameter estimates, determination coefficient $\left(\mathrm{R}^{2}\right), \mathrm{F}$ value, residual standard error (EPR) and variation coefficient $(\mathrm{CV} \%)$.

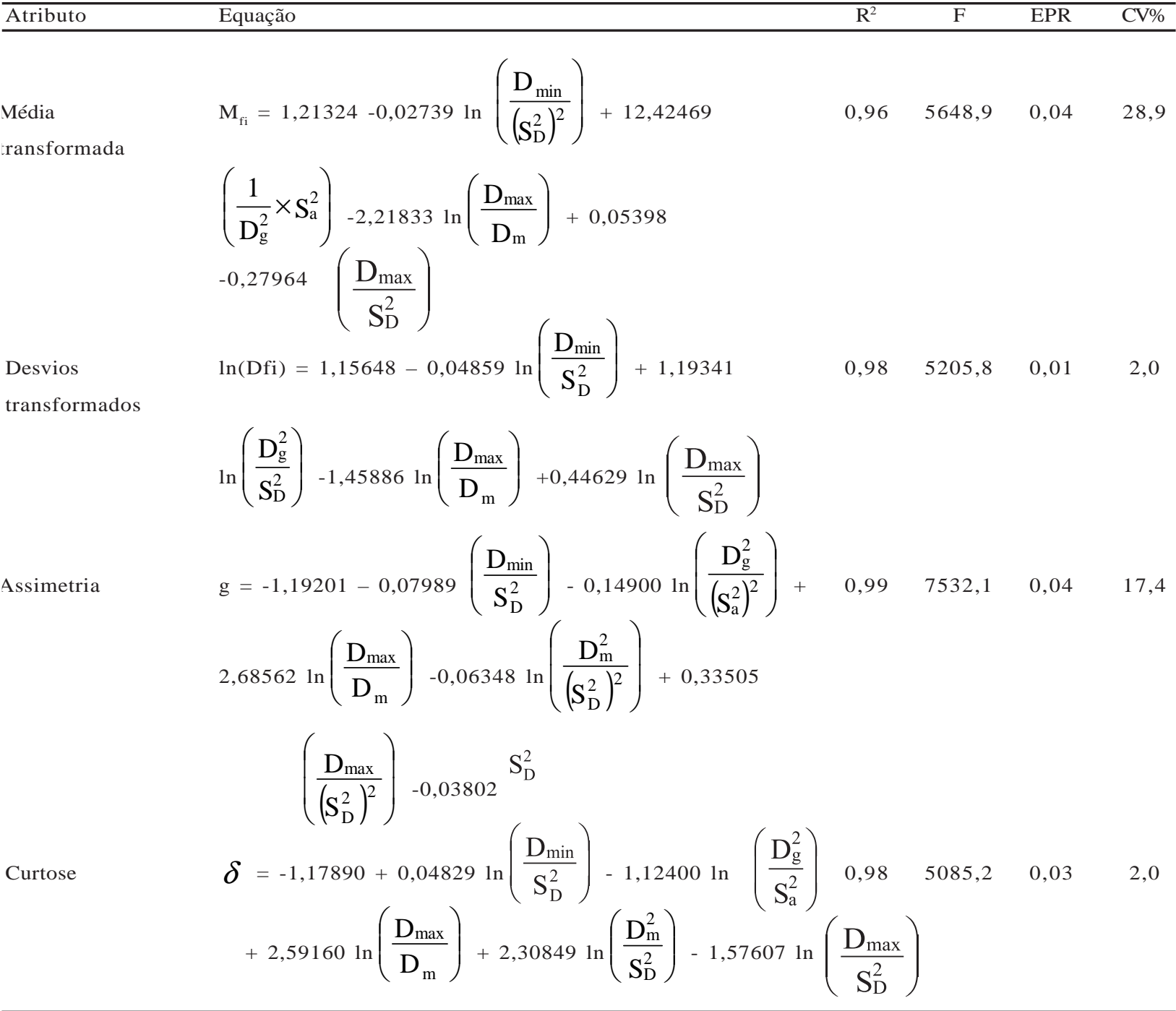

Em que: $\mathrm{M}_{\mathrm{fi}}=$ média transformadas das frequências dos diâmetros; $\mathrm{Dfi}$ = desvios transformados das frequências dos diâmetros; $\mathrm{g}=$ parâmetro assimetria; $\mathrm{d}$ = parâmetro curtose; $\mathrm{D}_{\min }=$ diâmetro mínimo aproveitável $(\mathrm{cm}) ; \mathrm{s}^{2}{ }_{\mathrm{D}}=$ variância dos diâmetros; $\mathrm{D}_{\mathrm{g}}=$ diâmetro médio quadrático $(\mathrm{cm}) ; \mathrm{D}_{\max }=$ diâmetro máximo $(\mathrm{cm}) ; \mathrm{e} \mathrm{D}_{\mathrm{m}}=$ média aritmética do diâmetro $(\mathrm{cm})$. 
A estratificação da floresta foi estabelecida em três classes de produtividade e por classe de idade. Os resultados encontrados dos valores médios da função $S_{B}$ por classe de produtividade estão apresentados na Tabela 3.
Convém ressaltar que, em ambos os casos, os resultados encontrados na classe de produtividade III não atendem à condição estatística adotada. Pelo teste estatístico aplicado, houve evidências para rejeitar

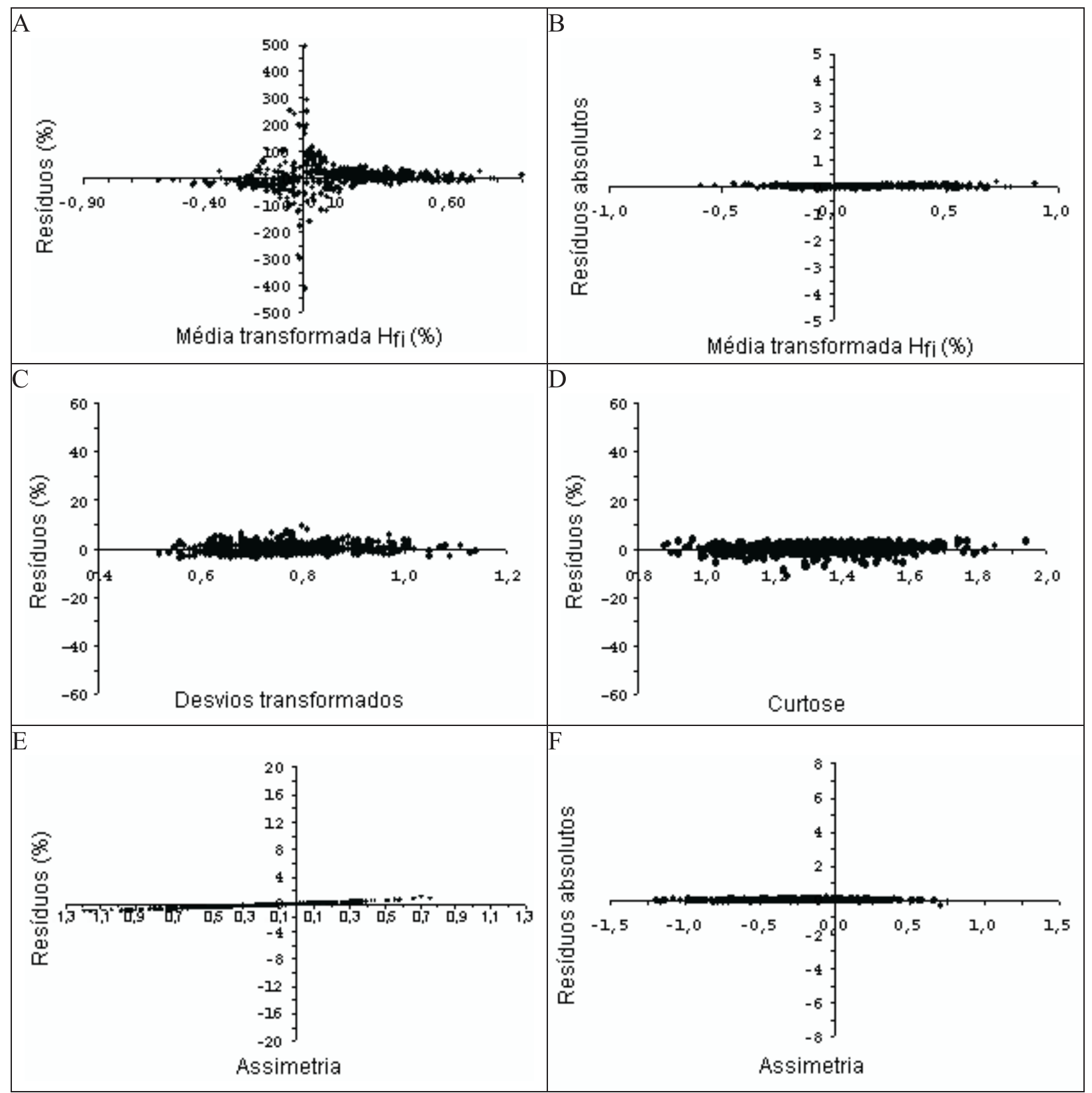

Figura 1 - Distribuição dos resíduos, absoluto e em porcentagem, da média transformada, desvios transformados, curtose e assimetria da função $S_{B}$ de Jonhson.

Figure 1 - Residue distribution, absolute and percentage values of the transformed mean, transformed deviations, asymmetry; kurtosis of the $\mathrm{S}_{\mathrm{B}}$ Jonhson's function.

R. Árvore, Viçosa-MG, v.33, n.5, p.853-863, 2009 
Tabela 3 - Prognose média do volume, número de árvores e área basal nas classes de produtividade I, II e III. Table 3 - Prediction of the volume, number of the individuals and basal area in productivity classes I, II and III.

\begin{tabular}{|c|c|c|c|c|c|c|c|c|c|}
\hline \multirow[t]{3}{*}{ Parâmetros } & \multicolumn{9}{|c|}{ Variáveis } \\
\hline & \multicolumn{3}{|c|}{ Volume } & \multicolumn{3}{|c|}{ Número de árvores } & \multicolumn{3}{|c|}{ Área basal } \\
\hline & III & II & $\bar{I}$ & III & II & I & III & II & $\bar{I}$ \\
\hline Média observada & 31,91 & 44,99 & 66,08 & 1293 & 1169 & 1151 & 5,96 & 7,55 & 9,20 \\
\hline Média estimada & 39,93 & 47,73 & 66,72 & 1258 & 1157 & 1141 & 7,21 & 7,93 & 9,09 \\
\hline Variância & 47,09 & 82,97 & 3,16 & 1884 & 1535 & 1440 & 0,77 & 1,61 & 0,62 \\
\hline Desvio padrão & 6,86 & 9,11 & 1,78 & 43 & 39 & 38 & 0,877 & 1,27 & 0,79 \\
\hline Desvios de prognose & $-7,48$ & $-2,74$ & $-0,63$ & 33 & 12 & 10 & $-1,17$ & $-0,38$ & 0,13 \\
\hline Coeficiente de variação (\%) & 21,51 & 20,25 & 2,68 & 3,36 & 3,35 & 3,29 & 14,74 & 16,78 & 7,82 \\
\hline Valor de $\mathrm{t}$ & $-4,08$ & $-1,31$ & $-1,60$ & 2,83 & 1,39 & 1,19 & $-4,98$ & $-1,31$ & 0,74 \\
\hline Valor de tc $(\mathrm{n}-1 ; \mathrm{a} 0,05)$ & 1,771 & 1,729 & 1,729 & 1,771 & 1,729 & 1,729 & 1,771 & 1,729 & 1,729 \\
\hline Nível de significância & $* *$ & NS & NS & $* *$ & NS & NS & $* *$ & NS & NS \\
\hline
\end{tabular}

a hipótese da nulidade, no nível a = 0,05 de probabilidade, pois a diferença entre os valores observados e estimados de todas as variáveis em estudo foi estatisticamente significativa

Diante dos resultados obtidos pela função $S_{\mathrm{B}}$ pode-se assegurar que, ambos os modelos podem ser utilizados em um sistema de prognose por classe de diâmetro sem prejuízo na eficiência e precisão dos modelos, bastando para isso que a base de dados seja bem controlada.

Na Figura 2, pode-se observar que o gráfico da função do volume estimado, o número de árvores e a área basal, segue o mesmo padrão dessas variáveis, que foram obtidos por ocasião do inventário. Os resultados de prognose obtidos pela função $S_{B}$ foram satisfatórios dentro do limite estatístico admissível de avaliação, contrastando com os resultados obtidos em nível de parcela.
Na classe de produtividade II, os resultados da prognose do volume, número de árvores e da área basal, obtido pela função $S_{B}$, são apresentados na Figura 3. Pode-se observar que, nessa classe de produtividade, os valores prognosticados estão bem próximos dos valores observados, contrastando com os resultados encontrados na classe de produtividade III, em que as diferenças dos valores estimados para os observados foram, pelo teste pareado, todos significativos.

A produção futura obtida na classe de produtividade I, ou seja, no sítio mais produtivo, está representada pela Figura 4, que mostra os valores do volume, o número de árvores e a área basal por hectare nas idades de prognose. Convém ressaltar que os dados não permitiram efetuar a prognose de cada índice de sítio, visto que o número de parcelas foi insuficiente em cada classe de idade nos respectivos sítios, razão que levou a agrupar os dados da floresta em três classes, como descrito anteriormente.

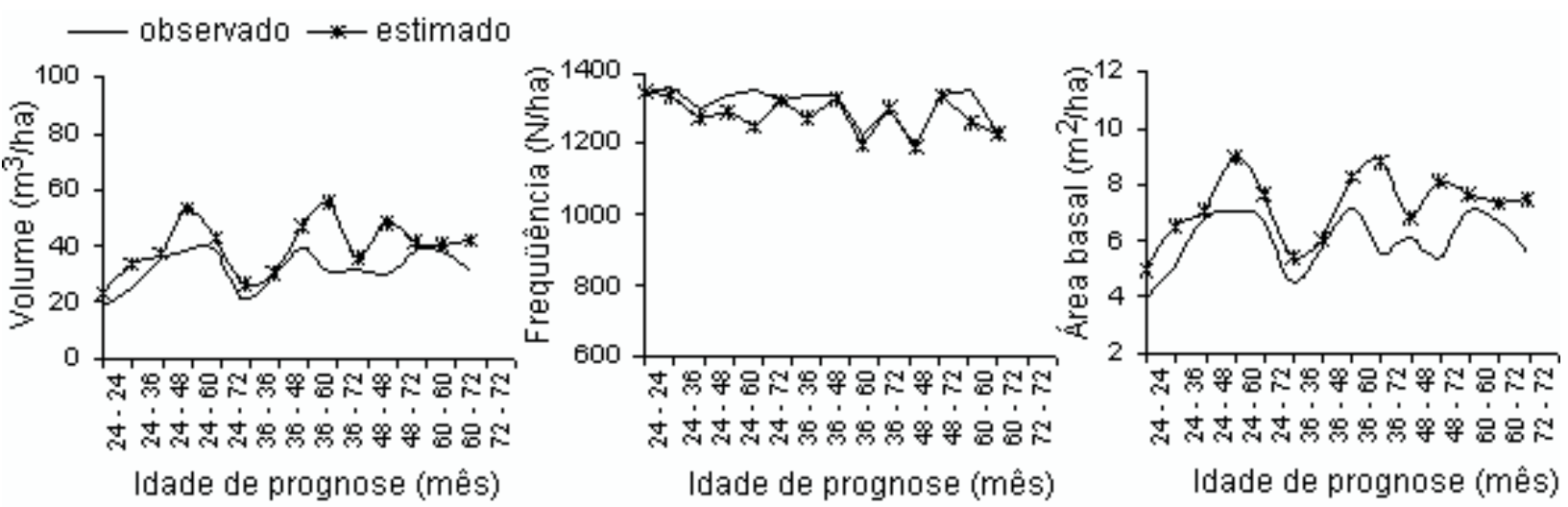

Figura 2 - Prognose da produção em volume, número de árvores e área basal na classe de produtividade III.

Figure 2 - Prediction of volume, number of individuals and basal area in productivity class III. 
Os resultados encontrados pelo sistema permitem afirmar que o modelo é apropriado para a projeção da produção futura do volume, do número de árvores e da área basal, visto que os valores prognosticados estão de acordo com os valores observados por ocasião das remedições. As diferenças entre os valores observados e estimados foram, pelo teste "t" pareado, não significativos para $\mathrm{a}=0,05$ de probabilidade.

Uma situação de menor precisão foi detectada na classe de produtividade III, com erros médios em porcentagens inferiores a $20 \%$, exceto para o volume. Nas classes de produtividade II e I, os valores encontrados são plenamente confiáveis, o que permite assegurar que, para fins de planejamento, o sistema é perfeitamente aceitável, tornando-se ferramenta importante ao gerente florestal.
Os resultados estão em conformidade com os encontrados por Guimarães (1994), quando aplicou modelos por classe de diâmetros em plantios de eucalipto no Estado de Minas Gerais. Da mesma maneira foram, também, compatíveis com os resultados encontrados por Cunha Neto et al. (1994), quando aplicou a função Weibull em modelo por classe de diâmetro para Eucalyptus grandis e Eucalyptus urophylla, e também por Scolforo e Thiersch (1998).

\subsection{Eficiência do sistema de prognose}

Os resultados da floresta sem estratificação foram, sistematicamente, tendenciosos, e, quando aplicado, o teste " $t$ " indicou para a rejeição da nulidade. Entretanto, quando o sistema de prognose foi aplicado aos dados estratificados por idade e classe de produtividade, os

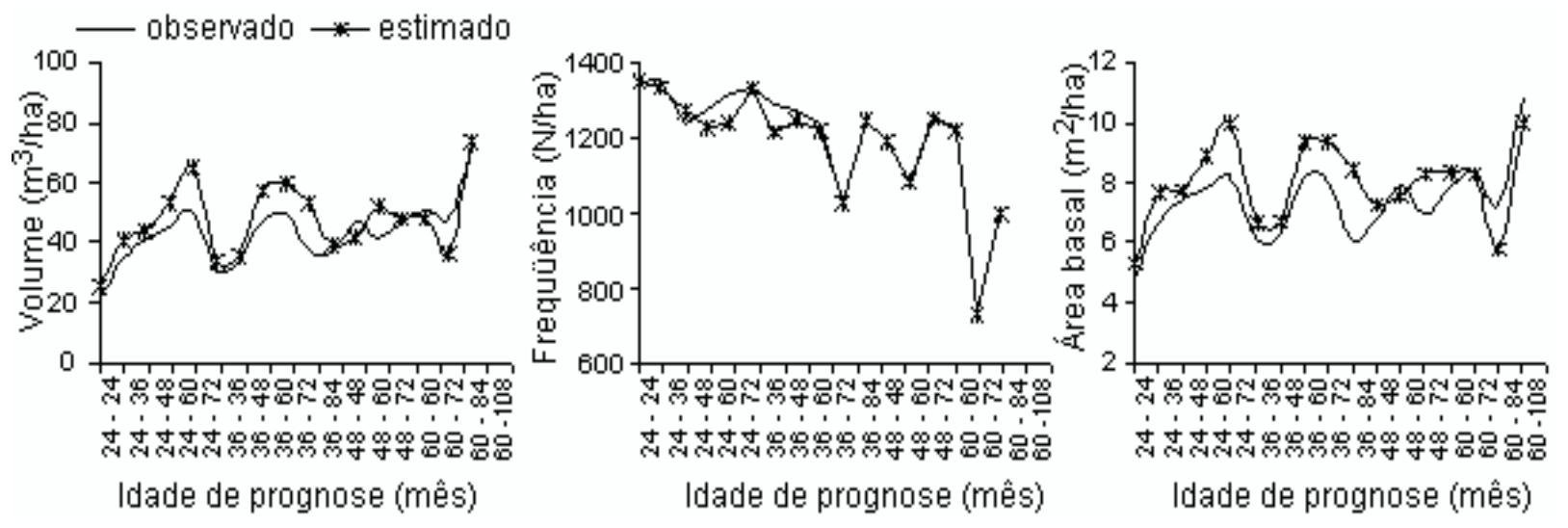

Figura 3 - Prognose da produção em volume, número de árvores e área basal na classe de produtividade II. Figure 3 - Prediction of volume, number of individuals and basal area in productivity class II.

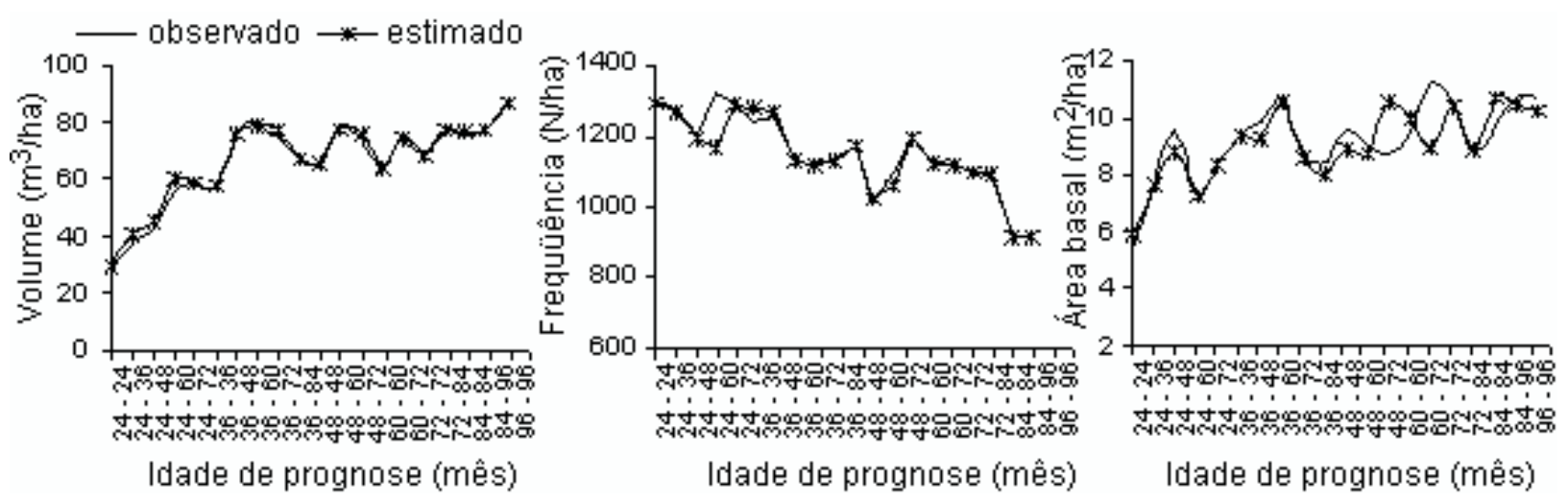

Figura 4 - Prognose da produção em volume, número de árvores e área basal na classe de produtividade I.

Figure 4- Prediction of volume, number of individuals and basal area in productivity class I. 

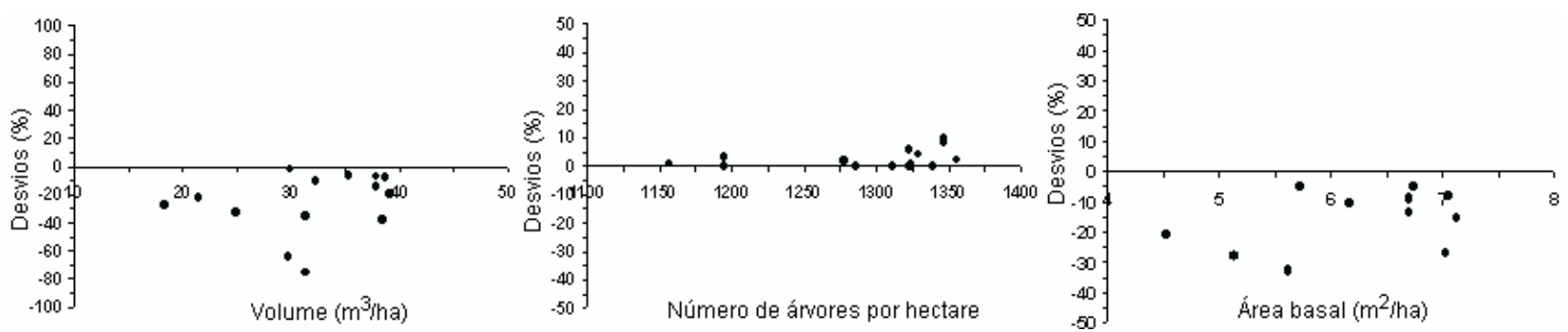

Figura 5 - Desvios de prognose do volume, número de árvores e área basal na classe de produtividade III. Figure 5 - Prediction deviations of volume, number of individuals and basal area in productivity class III.
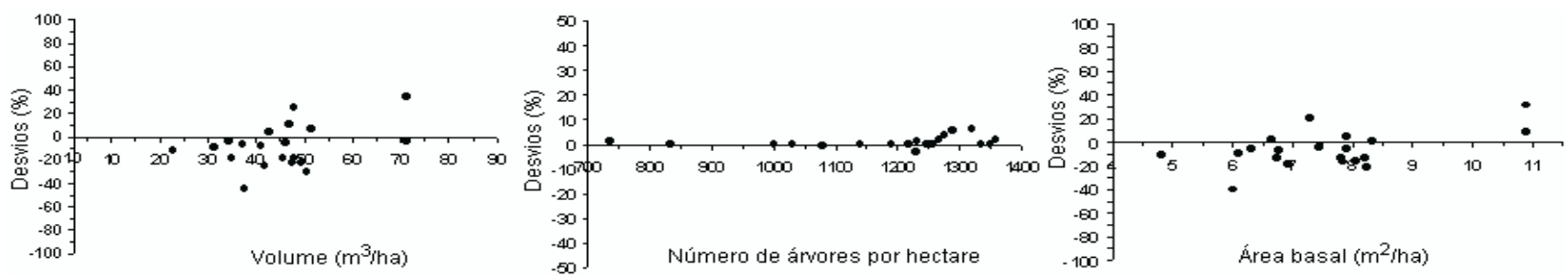

Figura 6 - Desvios de prognose do volume, número de árvores e área basal na classe de produtividade II. Figure 6 - Prediction deviations of volume, number of individuals and basal area in productivity class III.
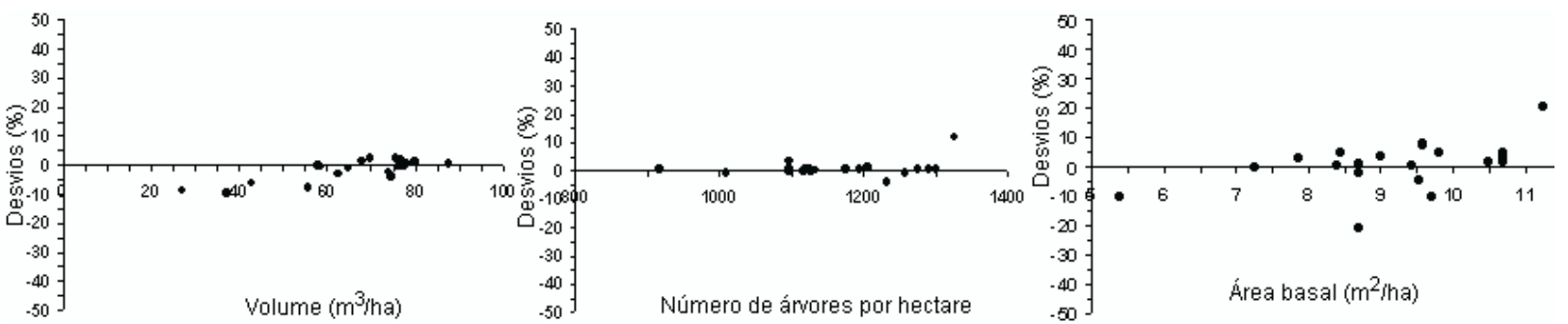

Figura 7 - Desvios de prognose do volume, número de árvores e área basal na classe de produtividade I.

Figure 7 - Prediction deviations of volume, number of individuals and basal area in productivity class III.

resultados foram considerados estatisticamente, adequados, exceto na classe de produtividade III, em que as diferenças entre os valores observados e estimados foram, estatisticamente, significativas. A Figura 5 permite visualizar os resultados dos desvios de prognose em porcentagem na classe de produtividade III.

Ao realizar o teste individualmente, par a par, apenas as prognoses efetuadas para as idades de 24 para 60 ; de 36 para 72; e de 48 para 72 meses, foram, entretando estatisticamente diferentes a uma probabilidade de $95 \%$, rejeitando-se a hipótese da nulidade. Ao testar a hipótese para $99 \%$, todos os resultados foram considerados não significativos, ou seja, houve evidências para aceitar a hipótese da nulidade. Esses resultados, embora apresentem padrão de tendência, permitem assegurar que o sistema é válido para prognosticar a produção em volume, número de árvores sobreviventes e área basal, por classe de diâmetro em determinada idade futura.

Os resultados obtidos na classe de produtividade II mostraram-se mais adequados quando comparados com os valores prognosticados na classe de produtividade III. Constatou-se que, nas idades de 24 para 72; de 36 para 84; e na idade de 104 meses, a diferença entre o volume observado e estimado não foram estatisticamente satisfatória, ou seja, houve evidência para rejeitar a hipótese de nulidade no nível de $95 \%$ de probabilidade.

A Figura 6 apresenta o comportamento dos desvios em porcentagem do volume, número de árvores e área basal nessa classe. Para o número de árvores e a área

R. Árvore, Viçosa-MG, v.33, n.5, p.853-863, 2009 
basal, o sistema de prognose também demonstrou ser eficiente, pois, diante do teste aplicado, constatou-se que apenas nas idades 24 a 72 e 36 a 48 meses foi rejeitada a hipótese da nulidade, fato esse que se repetiu com a área basal, dessa feita para as idades de 36 a 84 e 104.

Para a classe de produtividade I, após a aplicação do teste " $t$ " pôde-se verificar que os resultados foram considerados estatisticamente significativos nas idades de 24 para 36, 60 e 72 meses e, também, de 36 para 96 meses. Isso ocorreu, provavelmente, pela dificuldade no controle da base de dados e pelo número de amostras utilizadas em cada classe de idade. A prognose do número de árvores e da área basal teve resultados melhores na idade de 24 para 72 meses e para a área basal nas idades de 48 para 84 e de 60 para 72 meses, que foram estatisticamente significativos, sendo rejeitada a hipótese de nulidade. A Figura 7 permite observar o comportamento dos desvios de prognose para as variáveis em estudo.

$\mathrm{Na}$ avaliação dos resultados em gráficos da distribuição dos desvios de prognose em porcentagem, pode-se observar um padrão que foi considerado satisfatório para este estudo. Nas diferentes classes de produtividade, os resultados dos desvios seguiram padrão de comportamento tido como normal, resultado esse, que vem comprovar a validade do sistema; os dados não foram coletados com o propósito de desenvolver modelo de prognose por classe de diâmetro.

Diante de todos os resultados obtidos, foi possível observar claramente que a tendência da curva de prognose para o volume, número de árvores sobreviventes e a área basal é sempre a mesma nas parcelas permanentes ao longo do período da observação na floresta. Há maior ou menor afastamento entre o valor observado e estimado pelo sistema de prognose, o que permite afirmar que os efeitos das variáveis ambientais, tratamentos e, ainda, forma de coleta de dados influenciam os resultados. De maneira geral, de acordo com o teste estatístico aplicado, os resultados encontrados são válidos e estão plenamente compatíveis com os resultados obtidos por Cunha Neto et al. (1994), Guimarães (1994) e Scolforo e Thiersch (1998).

\section{CONCLUSÕES}

- A função Sb Johnson permite estimar a produção presente e futura de plantios homogêneos.
- A modelagem dos atributos do povoamento mostrou que os resultados obtidos foram satisfatórios dentro das condições estatísticas exigidas para prognose.

- O sistema desenvolvido constituiu instrumento adequado e confiável como auxiliar nas tomadas de decisões em manejo de florestas, proporcionando estimativas dentro dos níveis estatisticamente aceitáveis.

- O sistema de prognose desenvolvido permitiu fazer inferência sobre a produção presente e futura da floresta para idade e classe de produtividade, tendo resultados que foram satisfatórios dentro das condições estatísticas estabelecidas na análise.

\section{REFERÊNCIAS}

CAMPOS, J. C. C. Um sistema para estimar produção por classe de diâmetro e sua aplicação na interpretação de efeitos de desbastes. Árvore, v.5, n.1, p.1-16, 1981.

CAMPOS, J. C. C. et al. Aplicação de um modelo compatível de crescimento e produção de densidade variável em plantações de Eucalyptus grandis.

Árvore, v.10, n.2, p.121-134, 1986.

CUNHA NETO, F. R. et al. Modelo para predição da produção por classe de diâmetro para Eucalyptus grandis. Cerne, v.1, n.1, p.108-122, 1994.

GUIMARÃES, D. P. Desenvolvimento de um modelo de distribuição diamétrica de passo invariante para prognose e projeção da estrutura de povoamentos de eucalipto. 1994. 160f. Tese (Doutorado em Ciência Florestal) - Universidade Federal de Viçosa, Viçosa, MG, 1994.

LEITE, H. G. Ajuste de um modelo de estimação de frequiência e produção por classe de diâmetro, para povoamentos de Eucalyptus saligna Smith. 1990. 78f. Dissertação (Mestrado em Ciência Florestal) Universidade Federal de Viçosa, Viçosa, MG, 1990.

OLIVEIRA, E. B.; MACHADO, S. A. M.; FIGUEIREDO FILHO, A. Sistema para simulação do crescimento da produção de Pinus taeda L. e avaliação econômica de regimes de manejo. Árvore, v.22, n.1, p.99-111, 1998.

PÉLICO NETTO, S.; BRENA, D. A. Inventário florestal. Curitiba: [s.n.], 1993. 248p. 
SCOLFORO, J. R. S.; THIERSCH, A. Estimativas e testes da distribuição de frequiência diamétrica para Eucalyptus camaldulensis, através da distribuição SB, por diferentes métodos de ajuste. Scientia Forestalis, n.54, p.3-106, 1998.

SHIMIZU, J. Y.; KLEIN, H.; OLIVEIRA, J. R. V. Diagnóstico das plantações florestais em Mato Grosso. Cuiabá: Central de Texto, 2007. 63p
SCOLFORO, J. R. S.; MACHADO, S. A. Um sistema de crescimento e produção com simulador de desbaste. Scientia Forestalis, n.50, p.5164, 1996.

SILVA, V. S. M. Produção de Eucalyptus camaldulensis Dehnh. no Estado de Mato Grosso. 1999. 179f. Tese (Doutorado em Engenharia Florestal) - Universidade Federal do paraná, Curitiba, 1999. 